\title{
Le changement en 2013 !
}

\section{Changes in 2013!}

\section{Heresbach}

(C) Springer-Verlag France 2013

En 2006, la revue Acta Endoscopica est devenue la revue officielle de la SFED sous l'impulsion des différents présidents de cette société, notamment de Gérard Gay et de René Laugier. Les différents conseils d'administration ont dès cette date souhaité que cette revue soit dédiée à l'information professionnelle, tout en permettant la diffusion des présentations ou mises au point organisées par la SFED, notamment lors des JFHOD ou de Vidéo-Digest. Enfin, elle était également le lieu de publication des recommandations de type «Consensus en endoscopie » et « Fiches SFED ». Néanmoins, ces options n'excluaient pas la publication d'articles originaux ou de cas cliniques en langue française. Cette dualité n'a que partiellement fonctionné du fait d'un impact factor peu attractif et de la concurrence de revues européennes plus prestigieuses. Après quelques années de fonctionnement, la SFED devra, durant l'année 2013, se prononcer sur l'avenir qu'elle souhaite donner à cette revue. Dans l'attente de ces décisions, le comité de rédaction et le bureau de la SFED ont choisi de privilégier la mise en place de dossiers thématiques pour encore mieux se rapprocher de vos préoccupations tout en poursuivant la publication d'avis d'experts et de traduction des recommandations européennes en endoscopie digestive, en un mot vous tenir toujours mieux informés.
Cette nouvelle formule nécessite un nouveau souffle et c'est ainsi qu'après avoir assuré la fonction de rédacteur en chef pendant cinq ans dans un comité éditorial fortement épaulé par Christian Boustière et Gilles Lesur j'ai le plaisir de passer le témoin à ce dernier dont j'ai pu apprécier la constance et la ponctualité. C'est l'occasion pour moi de remercier ceux qui m'ont fait confiance pour cette mission et ceux qui ont contribué à la tenue d'une publication de qualité et, j'espère, attractive. Ce numéro est également pour moi l'occasion de vous faire part, à travers quelques news, des questions qui vont « encore » agiter notre profession, comme la prise en charge de polypes coliques minuscules et le diagnostic de leur possible nature adénomateuse sans analyse microscopique, la discussion autour du rythme de surveillances des patients après polypectomie, les données récentes concernant les recommandations sur la préparation à la coloscopie et l'épineuse question des indications des doubles actes endoscopiques. Il est encore tôt pour définitivement conclure sur ces sujets, mais le devoir d'un rédacteur est d'informer le plus fidèlement possible le lecteur de ces questions et de fournir les éléments de la littérature qui, à défaut de proposer des certitudes, peuvent aider à la réflexion. 